\title{
Antiserum from a Patient with Cerebellar Degeneration Identifies a Novel Protein in Purkinje Cells, Cortical Neurons, and Neuroectodermal Tumors
}

\author{
Robert B. Darnell, Henry M. Furneaux, and Jerome B. Posner \\ Department of Neurology and George C. Cotzias Laboratory of Neuro-Oncology, Memorial Sloan-Kettering Cancer \\ Center, New York, New York 10021 and Department of Neurology and Neuroscience, New York Hospital-Cornell \\ Medical Center, New York, New York 10021
}

The serum and cerebrospinal fluid of a patient (NB) with subacute cerebellar degeneration were found to contain a novel antineuronal autoantibody (anti-Nb). Using this antibody, we have identified and characterized antigens present in a subset of neurons in the CNS and in some neuroectodermal tumor lines. Anti-Nb antibody bound to antigens of $M_{r} 150,120$, and $65 \mathrm{kDa}$ in Western blots using extracts of human cerebellar Purkinje cells or human cerebral cortical neurons. Immunohistochemistry demonstrated relatively specific binding of anti-Nb IgG to Purkinje cells in sections of human cerebellum and to some neocortical neurons, especially those in layer VI. Because of the association of cerebellar degeneration with occult malignancies, we screened a number of tumor cell lines for immunoreactivity to anti- $\mathrm{Nb}$ antibody; only tumor lines of neuroectodermal origin (melanoma, small-cell lung cancer, and neuroblastoma) expressed the $\mathrm{Nb}$ antigen. Anti-Nb antibody thus identifies neuronal and tumor cell antigens that appear to be unique in size and distribution of expression.

Paraneoplastic neuronal syndromes are defined by the association of certain malignancies with neurologic disease in which the neuronal dysfunction cannot be attributed to a mass effect of the tumor itself (Anderson et al., 1987; Gulcher and Stefansson, 1988; Antel and Moumdjian, 1989; Vincent et al., 1989). A consistent observation in these disorders has been the finding of high titers of antineuronal antibodies in the serum and cerebrospinal fluid (CSF) of affected patients, which in some cases recognize those neurons in vitro that appear to be affected clinically. Several such antibodies have also been found to recognize antigens in tumor cells that are similar or identical to those recognized in neurons; these have been termed onconeural antigens.

Antisera from patients with paraneoplastic neurologic disease have been used to define a number of onconcural antigens. One such antineuronal antiserum, anti-Yo, is present in some pa-

\footnotetext{
Received May 28, 1990; revised Nov. 29, 1990; accepted Nov. 30, 1990.

We thank Drs. Fred Plum and Torsten Wiesel for critical reading of the manuscript, Dr. Steven Goldman for help with photomicrography, and members of the Cotzias laboratory, especially Dr. Josep Dalmau and Ellen Wong, for their assistance during this work. This research was supported in part by NIH Grant NS 26064 NINDS and by American Cancer Society Grant PDT-359-ACS.

Correspondence should be addressed to Jerome B. Posner, Department of Neurology, Memorial Sloan-Kettering Cancer Center, 1275 York Avenue, New York, NY 10021 .

Copyright (C) 1991 Society for Neuroscience $0270-6474 / 91 / 111224-07 \$ 03.00 / 0$
}

tients with paraneoplastic cerebellar degeneration and gynecologic cancer. Anti-Yo antiserum binds to two antigens of $M_{r} 62$ and $34 \mathrm{kDa}$ (Greenlee and Brashear, 1983; Jaeckle et al., 1985; Cunningham et al., 1986) that are expressed in both cerebellar Purkinje cells and, specifically, in breast or ovarian tumors isolated from these patients (Greenlee and Brashear, 1987; Furneaux et al., 1989; Dalmau et al., 1990). A second antineuronal autoantibody, termed anti-Hu, has been found in patients with a variety of neurologic dysfunctions, ranging from sensory neuropathy (Graus et al., 1985, 1986; Budde-Steffen et al., 1988a) to profound short-term memory loss (limbic encephalopathy; Graus et al., 1987); these patients invariably harbor small-cell lung cancers (SCLC). Anti-Hu antiserum binds to a nuclear antigen of $M_{r} 37 \mathrm{kDa}$ present in all neurons and expressed in high titer in SCLC tumors obtained from these patients (Graus et al., 1985, 1986; Dalmau et al., 1990). Anti-Ri is a third antineuronal antibody, associated with a neurologic syndrome of loss of eye and muscle control (termed paraneoplastic opsoclonus/myoclonus), that binds antigens of $M_{r} 55$ and $80 \mathrm{kDa}$ that are also expressed in breast carcinomas of patients with this syndrome (Budde-Steffen et al., 1988b; A. Luque, H. M. Furneaux, and J. B. Posner, unpublished observations). Taken together, these observations suggest a common pathogenesis for the paraneoplastic neuronal syndromes: an underlying malignancy provokes an immune response characterized by the production of an antitumor antibody that cross-reacts with neurons, and results, directly or indirectly, in neurologic disease.

Here, we describe the antigen specificity of a novel antineuronal antiserum (anti-Nb), identified in a patient with cerebellar degeneration, and compare it with the specificity of two previously characterized antineuronal antisera, anti-Yo and anti$\mathrm{Hu}$. We found that the anti-Nb antibody identifies a novel onconeural antigen with a unique distribution of expression and defines a clinical variant of subacute cerebellar degeneration.

Some of these results have been reported in abstract form (Darnell et al., 1989).

\section{Materials and Methods}

Human tissue. Human cerebellum or cerebral cortex was obtained at autopsy from neurologically normal patients with cancer, for use in immunohistochemistry and Western blot analysis. Purkinje and cerebral cortical neurons were purified according to the method of Yanagihara and Hamberger (1973), as modified by Cunningham et al. (1986). Tumor cell extracts were prepared by lysis of cell pellets in $0.1 \%$ NP40 and addition to SDS lysis buffer.

Immunohistochemistry. Serum was biotinylated by passing normal or patient sera over a protein-A Sepharose column, eluting with $\mathrm{Na}$ 
citrate (pH, 2.5) to purify IgG, dialysis overnight against PBS, and reaction with $50 \mu \mathrm{g}$ biotin $N$-hydroxysuccinimide ester (Vector Labs) for $2 \mathrm{hr}$ at room temperature, followed by dialysis against PBS. Control and experimental sera were biotinylated in tandem, using identical reaction conditions. Serial dilutions of each biotinylated IgG, dotted onto nitrocellulose and stained with avidin-biotin peroxidase complex (Vectastain ABC complex, Vector I abs; see below), revealed effective and commensurate biotinylation (per $\mu \mathrm{g}$ of $\mathrm{IgG}$ ) of normal and experimental samples (data not shown). To ensure that biotinylation did not alter the binding affinity of anti- $\mathrm{Nb} \mathrm{IgG}$, preincubation of tissue sections with native anti- $\mathrm{Nb}$ antiserum was shown to abolish completely subsequent binding of biotinylated anti-Nb IgG (data not shown).

Six-micron frozen sections were cut on a cryostat, fixed in cold acetone for $10 \mathrm{~min}$, and washed in PBS. Sections were treated in $0.3 \% \mathrm{H}_{2} \mathrm{O}_{2}$ at room temperature for $5 \mathrm{~min}$ to inactivate endogenous peroxidase activity, washed in PBS, and preincubated with normal human serum (NHS; diluted 1:10) for 10 min to suppress nonspecific binding. NHS was removed, biotinylated IgG was added to the appropriate dilution (in PBS with $10 \%$ NHS), and sections were incubated overnight at $4^{\circ} \mathrm{C}$. After washing with PBS, sections were incubated with avidin-biotin peroxidase complex (Vectastain ABC complex, Vector Labs) for $30 \mathrm{~min}$, washed in PBS, and developed for 2 min with $0.05 \%$ diaminobenzidine$\mathrm{HCl}$ (Hsu et al., 1981). Each finding reported was repeated in several independent experiments; identical staining patterns were observed when native anti- $\mathrm{Nb}$ serum or CSF were compared with each other or with biotinylated anti-Nb IgG (data not shown).

Western blots. Protein content was medsured by the method of Bradford (1976). Protein extracts were electrophoresed on 8\% SDS-PAGE and transferred to nitrocellulose as described by Towbin et al. (1979). Filters were blocked for $1 \mathrm{hr}$ in $5 \%$ milk in PBS and incubated with antibody at the appropriate dilution in TBST $[0.2 \mathrm{M} \mathrm{NaCl}, 0.1 \mathrm{M}$ Tris $\mathrm{HCl}(\mathrm{pH}, 8.0), 0.2 \%$ Triton $\mathrm{X}-100,1.0 \% \mathrm{BSA}$ ] overnight at $4^{\circ} \mathrm{C}$. After washing in TBST/SDS (TBST plus $0.02 \%$ SDS), filters were incubated with ${ }^{125} \mathrm{I}$-protein $\mathrm{A}(0.1 \mu \mathrm{Ci} / \mathrm{ml})$ for $1 \mathrm{hr}$ at room temperature in TBST, washed with TBST/SDS, and exposed to X-OMAT x-ray film. The findings reported were confirmed on multiple blots, with identical results.

Case report. A 35-yr-old woman (NB) was well until November 1987, when she developed dysarthria, vertigo, and ataxia over 1 week, which progressed over the next 2 months, leaving her bedridden. Neurologic examination in February 1988 revealed no cognitive impairment by bedside or formal neuropsychiatric testing, but showed coarse horizontal nystagmus with a rotatory component on lateral gaze to either side, vertical nystagmus on downward gaze, diffuse hyperreflexia with bilateral extensor plantar responses, titubation of the head and trunk at rest, and a severe and incapacitating truncal and appendicular ataxia following attempted movements. She was unable to sit, stand, feed, or wash herself. An extensive workup was unrevealing, including HIV and autoimmune serologies [except an increase in circulating immune complexes, $496 \mu \mathrm{g} / \mathrm{ml}$ (normal, 0-50)]; lumbar puncture (3 WBCs, 2 RBCs; glucose, 62; protein, 17; no oligoclonal bands); serial CSF cytologies; CT scans of the head, chest, abdomen, and pelvis; and an MRI of the head and cervical spine. There has been no change in her clinical condition to date.

\section{Results}

\section{Anti-Nb binding in the CNS: immunohistochemistry}

To define the binding specificity of the anti-Nb antiserum within the CNS, we performed immunohistochemistry using biotinylated anti-Nb IgG and compared the results with two antineuronal antibodies of defined specificity: anti- $\mathrm{Hu}$, which binds to the nuclei of all neurons in the CNS, and anti-Yo, which binds selectively to Purkinje cells in the cerebellum. Figure 1 illustrates the binding of these biotinylated IgGs to thin serial sections of human cerebellum. Anti-Nb IgG (Fig. $1 A$ ) binds within the cerebellum to antigens that are prominently expressed in Purkinje cells; the specificity of this binding appears to be intermediate to that of the anti-Yo IgG (Fig. $1 B$ ) and anti-Hu IgG (Fig. $1 C$ ). No reactivity was observed with biotinylated normal IgG (Fig. 1D). Staining similar to that shown in Figure $1 A$ was seen with native anti-Nb antiserum at dilutions of up to 1:5000 and with anti-Nb CSF at dilutions of 1:100 (data not shown).

The binding specificity of anti-Nb IgG is shown in greater detail in Figure 2, a higher magnification of the staining of anti$\mathrm{Nb}$ (Fig. $2 A$ ) and anti-Hu IgG (Fig. $2 B$ ) to the cerebellum. Anti$\mathrm{Nb}$ IgG reacted intensely with a cytoplasmic or membrane component of Purkinje cells (Fig. $2 A$ ); the nucleus appeared spared, with the exception of the nucleolus. This staining pattern was different from that seen with anti-Hu IgG (Fig. $2 B$ ), which reacts with the nuclei of all neurons, or anti-Yo $\mathrm{IgG}$, which has a distinct 'tigroid' cytoplasmic staining pattern in Purkinje cells (not shown; see Jaeckle et al., 1985; Cunningham et al., 1986). Little or no other cellular staining was seen with anti- $\mathrm{Nb} \operatorname{IgG}$, with the exception of very rare intensely reactive cells in the granular and molecular layers (not shown) and a low level of diffuse staining throughout the gray matter in sections of cerebellum (Fig. $1 A$ ) and cerebral cortex (see below).

Figure 3 compares the cortical staining of these antibodies. Anti-Nb IgG bound to cortical neurons (Fig. $3 \mathrm{~A}$ ); essentially no staining was evident in white matter or glial cells (evident from counterstained sections; not shown). At higher power (Fig. 3D), the cellular elements stained are seen to be cortical neurons, with immunoreactivity in the cytoplasm and nucleolus, sparing the nucleus, as was seen in Purkinje cell neurons. A low level of diffuse staining to an extracellular component of the gray matter (cf. anti-Hu IgG staining, Fig. $3 B$ ) was evident. No staining was seen with either anti-Yo IgG (not shown) or normal human IgG (Fig. 3C).

The pattern of anti- $\mathrm{Nb}$ binding within the six layers of the cerebral cortex suggested a gradient of neuronal binding. A greater proportion of neurons exhibited intense immunoreactivity with anti-Nb IgG in the deep layers of the gray matter than in the more superficial layers (cf. layer VI and layer I, Fig. 3A). By contrast, serial sections of cortex reacted with anti-Hu IgG (Fig. $3 B$ ) showed no gradient of neuronal binding. While difficult to quantitate accurately with this methodology (exacerbated by the high background staining seen with anti- $\mathrm{Nb}$ ), counts of neurons immunoreactive with anti-Nb IgG relative to neurons reactive with anti-Hu IgG suggested that anti-Nb IgG bound to approximately $90-100 \%$ of layer VI neurons, $50-60 \%$ of layer IV neurons, and 15-20\% of layer I neurons. Neurons that did react with anti- $\mathrm{Nb}$ in the superficial cortical layers appeared to stain less intensely than those in deeper layers, perhaps suggesting a lower quantitative expression of antigen in these neurons.

\section{Anti-Nb binding in the CNS: Western blots}

Figure 4 illustrates a Western blot of extracts of human cerebellar Purkinje cells and human cerebral cortex reacted with anti- $\mathrm{Nb}$ antiserum. Three bands of $M_{r} 150,120$, and $65 \mathrm{kDa}$ were identified; they comigrated precisely in both extracts. These results suggest that the same antigen(s) recognized by anti-Nb antiserum in Purkinje cell extracts is also expressed in cerebral cortical cells. Serial dilutions of patient NB's serum and CSF (data not shown) demonstrated reactivity with these bands at dilutions of greater than $1: 1000$ in the serum and 1:100 in CSF. No immunoreactivity was observed when normal human serum (Fig. 4, NHS) was blotted with either Purkinje or cortical cell extracts.

The Purkinje cell antigens recognized by anti- $\mathrm{Nb}$ antiserum [collectively termed "NB-150" protein(s)] were distinct in molecular weight from previously detected anti-human Purkinje cell autoantibodies, including anti-Yo (Fig. 4, Yo; Cunningham 

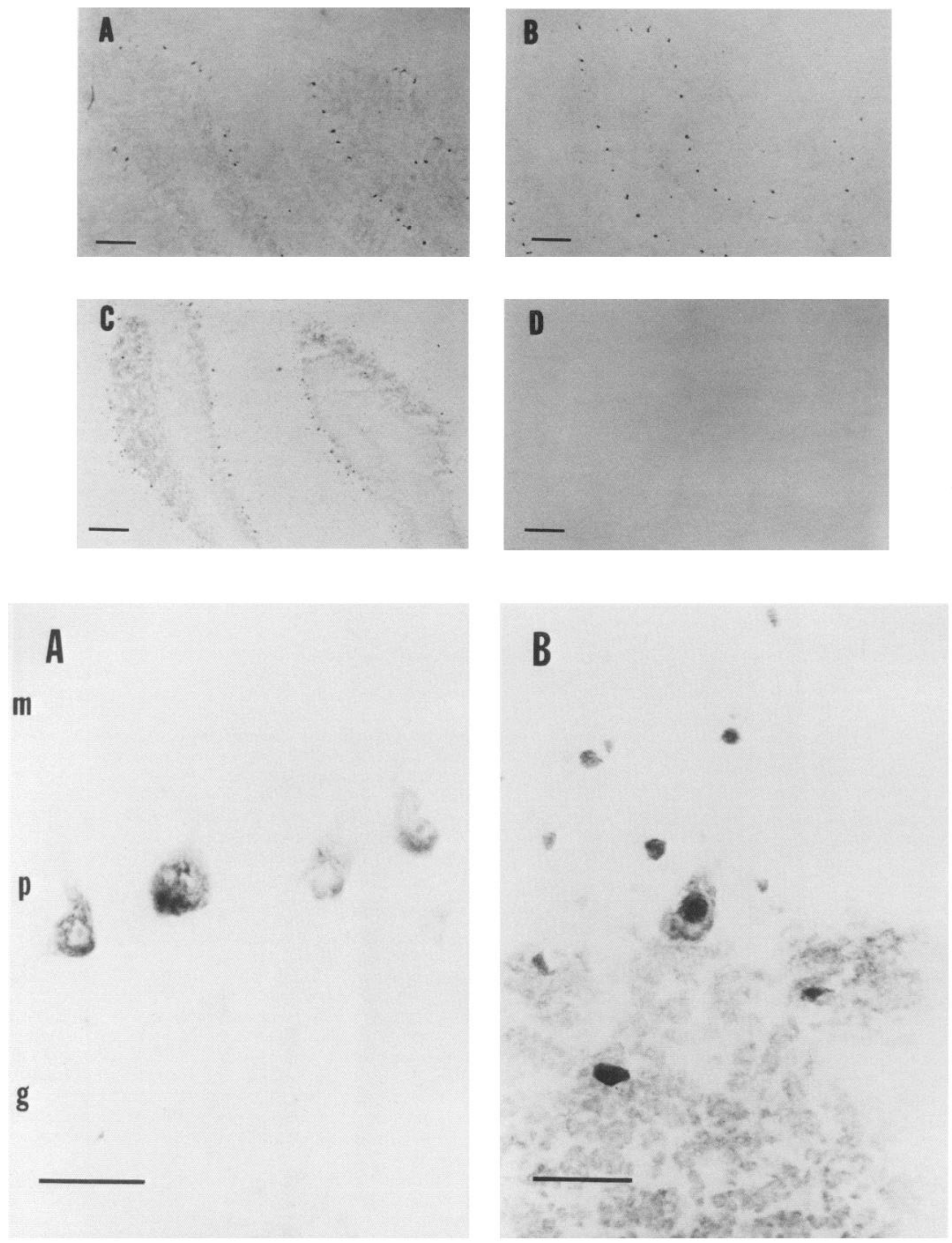

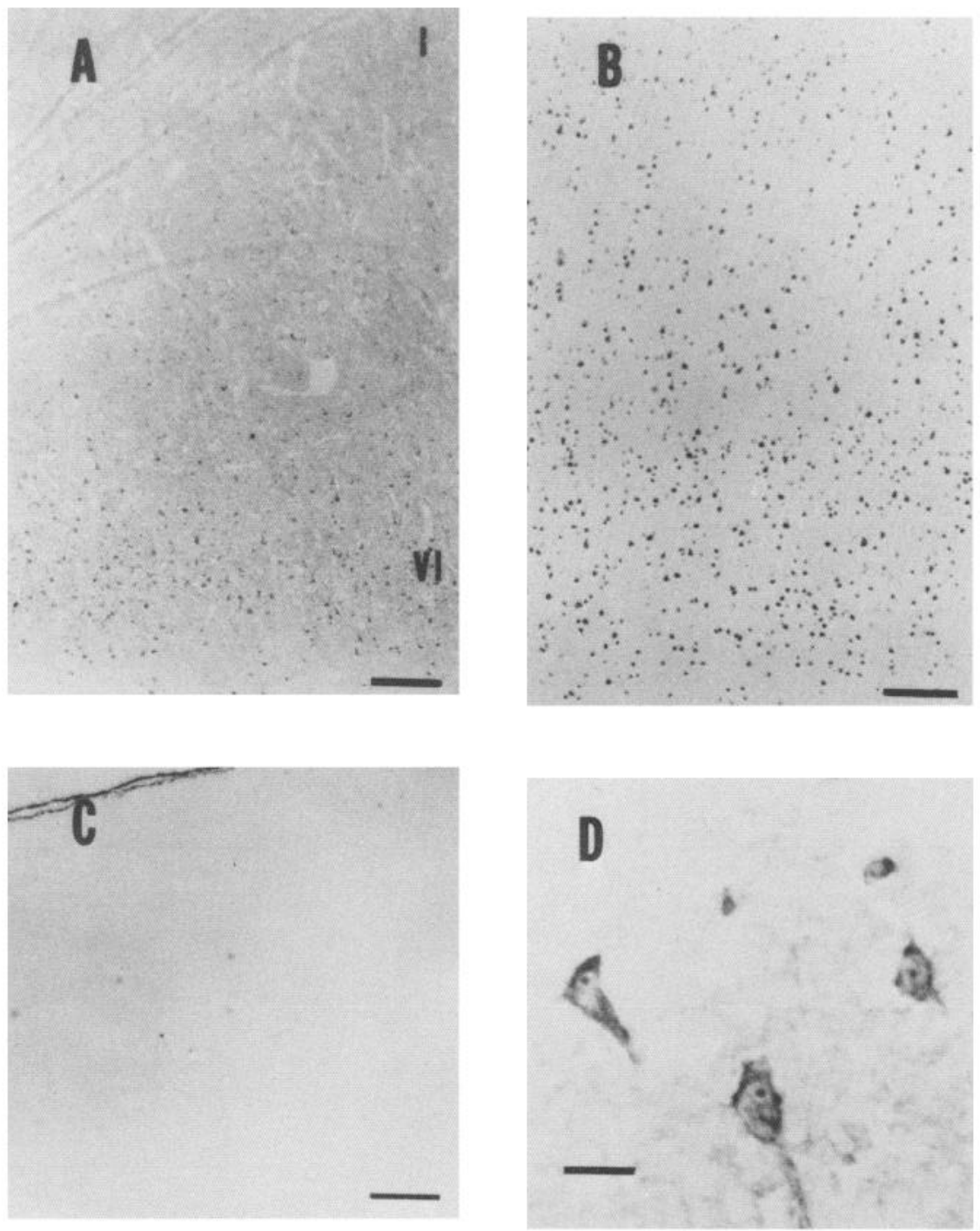

Figure 3. Anti-Nb IgG reacts with cortical neurons of layer VI: immunohistochemistry. Serial frozen sections of normal human cerebral cortex were incubated with biotinylated anti-Nb IgG $(100 \mu \mathrm{g} / \mathrm{ml} ; A)$, biotinylated anti-Hu IgG $(50 \mu \mathrm{g} / \mathrm{ml} ; B)$, or biotinylated normal human IgG $(200 \mu \mathrm{g} / \mathrm{ml} ; C)$. Sections were not counterstained, and photographs were taken at $40 \times$ power. $A$, Anti-Nb IgG binds to some cortical neurons, with a predominance of staining in layer $V I$ (cf. staining in layer $I$ ). $B$, A serial section developed together from slide used in $A$, but reacted with anti-Hu IgG. The photograph is oriented as in $A$ and demonstrates equal intensity reactivity of anti-Hu with neurons in layers I-VI; the laminar pattern of the cortex can be appreciated. $C$, Normal human $\mathrm{IgG}$ is not reactive (the edge of the tissue section, which stained nonspecifically, is shown). $D, A$ high-power photograph of the cortical cellular elements stained with anti-Nb IgG illustrated in $A$. Immunoreactivity to a cytoplasmic or membrane component of cortical neurons is evident and, to a lesser degree, to a component of the background matrix. Scale bars: $A-C, 375 \mu \mathrm{m} ; D, 25 \mu \mathrm{m}$. et al., 1986), anti-Hu (Fig. 4, Hu; Graus et al., 1985), anti-Ri (data not shown; see Budde-Steffen et al., 1988b), and others (Tanaka et al., 1986; Anderson et al., 1988a,b; Tsukamoto et al., 1989). Figure 4 also demonstrates the specific nature of the Purkinje and cortical cell extracts: anti-Yo antiserum reacted with Purkinje but not cortical extracts (Cunningham et al., 1986), while anti-Hu antiserum reacted with both.

\section{Anti-Nb binding in neuroectodermal tumor cell lines}

Because many patients with cerebellar degeneration and autoantibodies manifest malignancies within several years after the onset of their neurologic syndrome (Anderson et al., 1987), we were particularly interested in the possibility that NB-150 might be expressed in tumor cells. Figure 5 shows the results of West-

Figure 1. Anti-Nb IgG reacts with human Purkinje cells: immunohistochemistry. Serial frozen sections of human cerebellum were incubated with biotinylated IgG and developed as described in Materials and Methods; initially, sections were not counterstained. $A$, Anti-Nb IgG (100 $\mu \mathrm{g} / \mathrm{ml})$ demonstrates staining predominantly to Purkinje cells, seen as small neurons outlining cerebellar folia. $B$, Anti-Yo IgG (50 $\mu \mathrm{g} / \mathrm{ml})$, which stains only Purkinje cell neurons in the cerebellum (Cunningham et al., 1986). C, Anti-Hu IgG (50 $\mu \mathrm{g} / \mathrm{ml})$, which stains all neurons in the granular, Purkinje, and molecular layers (Graus et al., 1985). D, Normal human IgG (200 $\mu \mathrm{g} / \mathrm{ml})$. Counterstaining each section (not shown) confirmed the integrity of each section and revealed that anti-Nb IgG bound to all the Purkinje cells present in each section. Scale bars, $375 \mu \mathrm{m}$.

Figure 2. The binding specificity of anti-Nb IgG $(A)$ compared with anti-Hu IgG $(B)$. To illustrate the cellular elements stained in Figure $1, A$ and $C$, photographs were taken at higher power from the same slides shown in Figure 1 . Two serial sections of cerebellum on a single slide were incubated with anti-Nb IgG $(A)$ or anti-Hu $\operatorname{IgG}(B)$ and developed together. The granular layer $(g)$, Purkinje layer $(p)$, and molecular layer $(m)$ are labeled in the photograph. Anti- $\mathrm{Nb} \operatorname{IgG}(A)$ reacts with the cytoplasm and nucleoli of all Purkinje cells, while anti-Hu IgG $(B)$ reacts with the nuclei of all neurons, sparing the nucleolus. Scale bars, $25 \mu \mathrm{m}$. 
Figure 4. Western blot analysis of antigens recognized by anti-Nb serum. Purkinje cell extracts were run on an $8 \%$ SDS polyacrylamide gel and reacted to a variety of patient NB's antisera. Approximately $50 \mu \mathrm{g}$ of Purkinje cell extract (Purkinje) or cortical extract (Cortex) was loaded per lane, blotted to nitrocellulose, and incubated with various antisera: $N H S$, normal human serum 1:500; $\mathrm{Nb}$, anti- $\mathrm{Nb}$ antiserum 1: $1000 ; Y o$, anti-Yo antiserum 1:1000; $H u$, anti-Hu antiserum 1:1000. Molecular-weight markers are indicated on the left. Anti-Nb antiserum recognizes 3 bands of approximate $M, 150,120$, and $65 \mathrm{kDa}$ in both Purkinje cells and cortex. Anti-Yo antiserum recognizes an antigen of $M_{r} 62 \mathrm{kDa}$ (and $34 \mathrm{kDa}$; not visualized here) in Purkinje but not cortical extracts (Cunningham et al., 1986). Anti-Hu antiserum recognizes an antigen of approximate $M_{r} 37 \mathrm{kDa}$ in both Purkinje and cortical extracts (Graus et al., 1985). There is no reactivity with NHS.

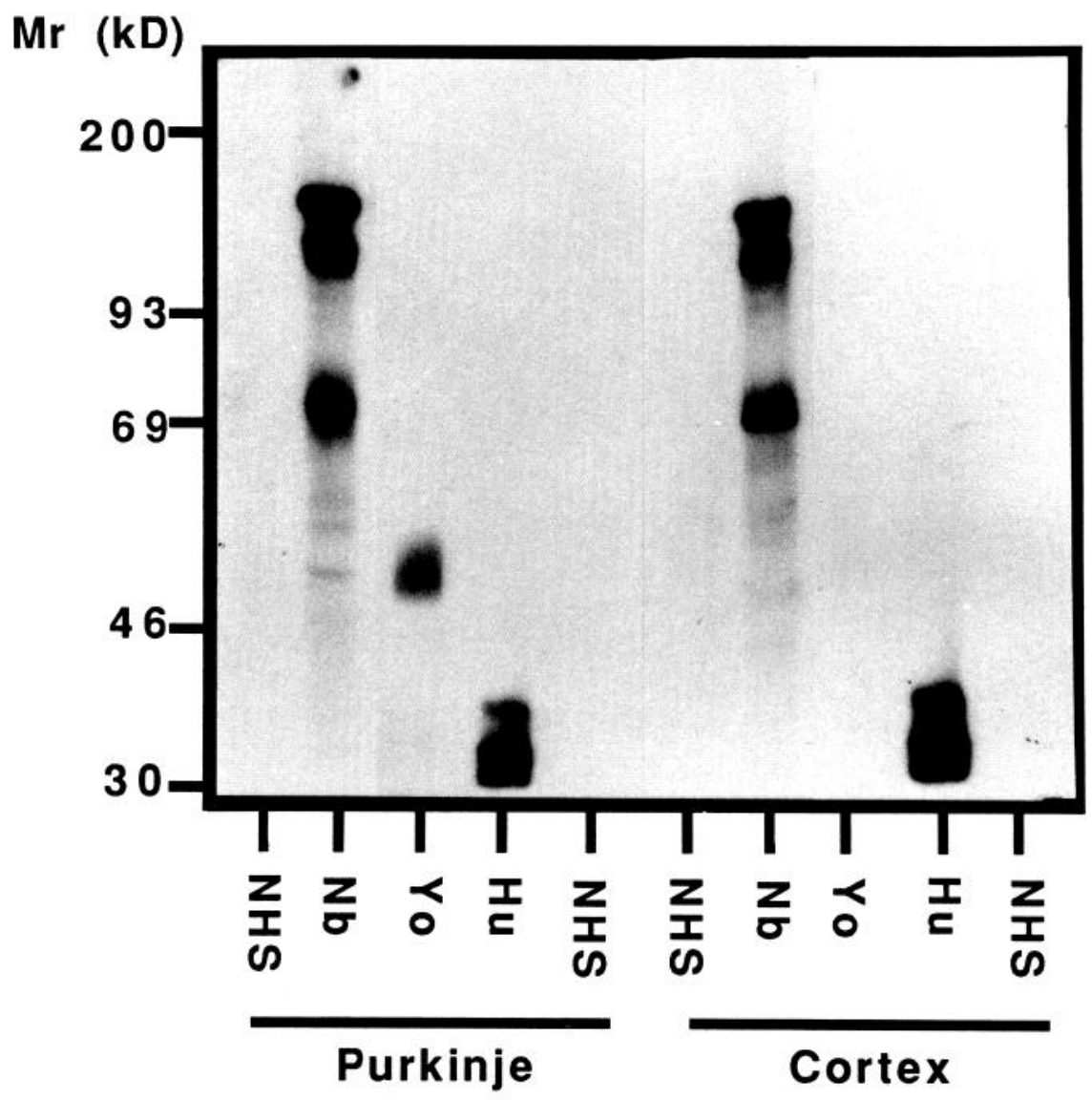

ern blots in which extracts of tumor cell lines were run on SDSPAGE and probed with anti-Nb CSF. Extracts from a number of tumor cell lines of neuroectodermal origin (melanoma, SCLC, and neuroblastoma) were immunoreactive with anti-Nb CSF; these results were repeated with two independent neuroblastoma cell lines and one independent melanoma cell line (data not shown). Several other tumor cell extracts were not reactive with anti-Nb CSF, including HeLa cells and breast and ovarian carcinoma cell lines, shown in Figure 5, and renal, uterine, and non-small-cell lung carcinoma cell lines (data not shown). Normal tissues were examined for immunoreactivity to anti- $\mathrm{Nb}$ antisera; lung, liver, ovary, adrenal, and thyroid were not reactive with anti-Nb by Western blot and/or immunohistochemistry (data not shown).

The antigen identified in neuroectodermal tumor cell extracts migrated at precisely the same molecular weight as the NB-150 protein from Purkinje cells (Fig. 5), suggesting that the NB-150 protein is expressed in neuroectodermal tumor cell lines in addition to Purkinje and some cerebral cortical neurons. While protein blots of tumor extracts demonstrate immunoreactivity predominantly with the $M_{r} 150 \mathrm{kDa}$ band, in each case longer exposures revealed the presence of the 120- and 65-kDa bands. Because the proportion of each band was found to vary between different preparations of extract from the same tumor cell lines, the lower-molecular-weight bands identified by anti-Nb antiserum may represent proteolytic fragments of the NB-150 antigen.

\section{Discussion}

We have found that antiserum from a patient with cerebellar degeneration recognizes a $150-\mathrm{kDa}$ protein in cerebellar Purkinje cell neurons, cerebral cortical neurons, and neuroectodermal tumor cell lines. Immunohistochemistry using anti- $\mathrm{Nb}$ antiserum demonstrates reactivity with Purkinje cell neurons and neocortical neurons, predominantly those of layer VI. Despite the comigration of $150-\mathrm{kDa}$ bands on Western blots of neuronal are identical to each other or to those recognized immunohistochemically, because the anti-Nb antibody is polyclonal. Such assignments can be made definitively only with monospecific reagents: monoclonal antibodies or cDNAs. Efforts to affinity purify the anti-Nb antibody with the $150-\mathrm{kDa}$ protein band have been unsuccessful (R. B. Darnell, H. M. Furneaux, and J. B. Posner, unpublished observations). Nonetheless, our data do suggest that the anti- $\mathrm{Nb}$ antisera recognize one or more neuronal and neuroectodermal antigens distinct from those previously detected with antineuronal autoantibodies (Graus et al., 1985; Cunningham et al., 1986; Tanaka et al., 1986; Anderson et al., 1988a,b; Budde-Steffen et al., 1988a,b; Tsukamoto et al., 1989), and that these proteins exhibit unusual patterns of expression in the CNS.

It has not generally been possible to demonstrate a strict correlation between neurons affected clinically in paraneoplastic disease and specificity of the associated antineuronal autoanand tumor extracts, we have not established that these antigens 


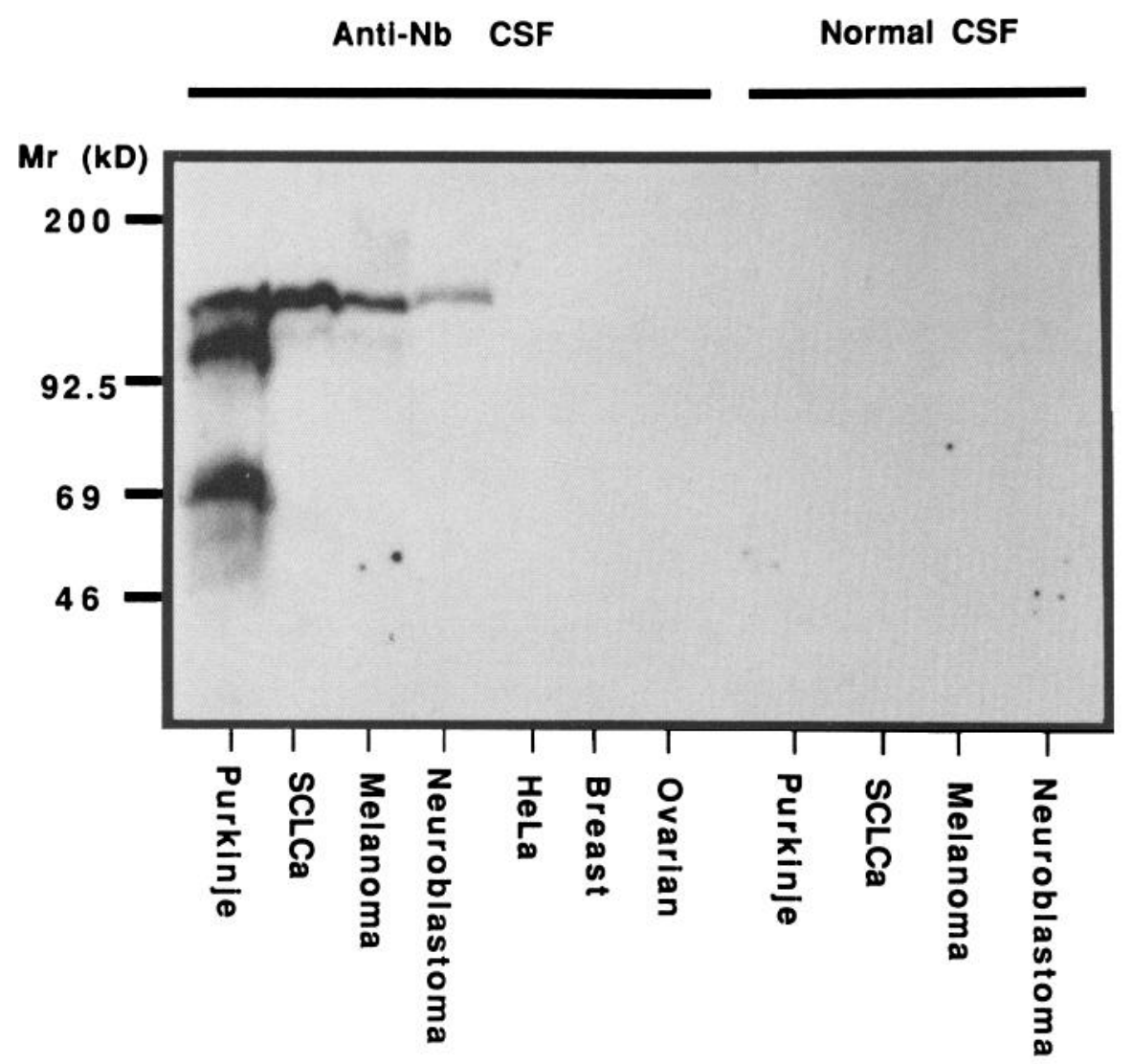

Figure 5. Anti-Nb antiserum reacts with neuroectodermal tumor lines: Western blot. In each lane, $20 \mu \mathrm{g}$ of protein extracts from Purkinje cells or a variety of tumor cell lines (as indicated) was run on $8 \%$ SDS polyacrylamide gels and incubated with either anti-NbCSF (1:100) or normal CSF $(1$ : $100)$. CSF gave the same results as anti$\mathrm{Nb}$ antiserum, but often with less nonspecific binding. Reactivity to anti- $\mathrm{Nb}$ CSF is seen with SCLC, melanoma, and neuroblastoma tumor lines, but not with $\mathrm{HeLa}$, breast, or ovarian tumor lines. No reactivity is seen with normal CSF. tibodies. For example, anti-Hu (Graus et al., 1986) and anti-Ri (Budde-Steffen et al., 1988b) antibodies each bind to all neurons of the CNS, yet are associated with discrete neurologic syndromes; anti-Yo, while binding specifically to Purkinje cells in the cerebellum, shows immunoreactivity with subsets of neurons in the cortex and brainstem (Greenlee et al., 1988; J. Henson, H. M. Furneaux, and J. B. Posner, unpublished observations). Whether the clinical specificity of the paraneoplastic neurologic syndromes relates to differential neuronal susceptibilities to autoantibodies, secondary levels of specificity, such as the involvement of $\mathrm{T}$ cells in the disease, or are independent of the presence of antineuronal antibodies is uncertain. Nonetheless, these observations do suggest that the Purkinje "plus" specificity of anti-Nb antibody need not mitigate against a role for this autoantibody in the pathogenesis of patient NB's cerebellar degeneration.

The observation that the same NB-150 protein may be coordinately expressed in subsets of neurons and neuroectodermal tumor cell lines parallels the observation that the paraneoplastic neurologic syndromes are associated with autoantibodies that bind to both neuronal and tumor cell antigens. Although we have not detected a malignancy in patient NB, $40-60 \%$ of patients ultimately diagnosed as having paraneoplastic neurologic disease develop neurologic dysfunction prior to the discovery of their malignancy (Greenlee and Brashear, 1983; Anderson et al., 1987). Several patients in whom no malignancy has been detectable clinically have turned out to harbor occult foci of neoplastic cells at autopsy or exploratory laparotomy (Anderson et al., 1988a,b). We continue to follow patient NB closely for a malignancy, particularly one of neuroectodermal origin.
The anti- $\mathrm{Nb}$ antibody appears to bind prominently to the cerebral cortical neurons of layer VI and a diminishing gradient of neurons in more superficial layers. There is no known clinical role specifically assigned to these neurons; in patient NB, examination of cortical function was unrevealing. Experimentally, layer VI consists of a diverse group of neurons with a variety of local, cortical, and subcortical projections and physiologic properties (Gilbert and Wiesel, 1979; Katz, 1987; Bolz et al., 1989); whether anti- $\mathrm{Nb}$ discriminates a subset of these neurons is unclear. While the projections from layer VI are diverse, and probably differ in various cortical areas, they are prominently involved in motor outflow, including outflow to the cerebellum (via the pons; Carpenter, 1976). Whether this binding of anti$\mathrm{Nb}$ antibody to layer VI cortical neurons contributed to the severity of the motor dysfunction seen clinically was unclear; the patient's complete pancerebellar syndrome could not be distinguished clinically from that seen in patients with "pure" Purkinje cell degeneration (anti-Yo syndrome).

Understanding the molecular mechanism by which neurons and tumor cells co-express shared antigens will depend on a molecular identification and analysis of genes that are expressed in each. There are several possibilities for the shared expression of onconeural antigens such as NB-150 in neurons and tumor cells: the "random" activation of neural antigens in tumor cell lines, the coordinate regulation of tumor genes and neural antigens by regulatory elements common to both cell types, or a functional role of a neural antigen in the selected phenotype of tumor cells. The distinction between these possibilities will depend on further characterization of the structure, function, and regulation of these interesting proteins. 


\section{References}

Anderson NE, Cunningham JM, Posner JB (1987) Autoimmune pathogenesis of paraneoplastic neurological syndromes. CRC Crit Rev Clin Neurobiol 3:245-299.

Anderson NE, Budde-Steffen C, Wiley RG, Thurman L, Rosenblum MK, Nadeau SE, Posner JB (1988a) A variant of the anti-Purkinje cell antibody in a patient with paraneoplastic cerebellar degeneration. Neurology 38:1018-1026.

Anderson NE, Rosenblum MK, Posner JB (1988b) Paraneoplastic cerebellar degeneration: clinical-immunological correlations. Ann Neurol 24:559-567.

Antel JP, Moumdjian R (1989) Paraneoplastic syndromes: a role for the immune system. J Neurol 236:1-3.

Bolz J, Gilbert CD, Wiesel TN (1989) Pharmacological analysis of cortical circuitry. Trends Neurosci 12:292-296.

Bradford M (1976) A simple method for protein determination. Ann Biochem 72:248.

Budde-Steffen C, Anderson NK, Rosenblum MK, Posner JB (1988a) Expression of an antigen in small cell lung carcinoma lines detected by antibodies from patients with paraneoplastic dorsal root ganglionopathy. Cancer Res 48:430-434.

Budde-Steffen C, Anderson NK, Rosenblum MK, Ford D, Wray SH, Posner JB (1988b) An antineuronal autoantibody in paraneoplastic opsoclonus. Ann Neurol 23:528-531.

Carpenter MB (1976) Human neuroanatomy. Baltimore: Williams and Wilkins.

Cunningham J, Graus F, Anderson N, Posner JB (1986) Partial characterization of the Purkinje cell antigens in paraneoplastic cerebellar degeneration. Neurology 36:1163-1168.

Dalmau J, Furneaux HM, Gralla RJ, Kris MG, Posner JB (1990) Detection of the anti-Hu antibody in the serum of patients with small cell lung cancer-a quantitative Western blot analysis. Ann Neurol 27:544-552.

Darnell RB, Furneaux H, Posner JB (1989) Characterization of antigens bound by CSF and serum of a patient with cerebellar degeneration: co-expression in Purkinje cells and tumor lines of neuroectodermal origin. Neurology [Suppl] 39:385.

Furneaux HE, Rosenblum M, Wong E, Woodruff P, Posner J, Graus F (1989) Selective expression of Purkinje neuron antigens in ovarian and breast tumors of patients with paraneoplastic cerebellar degeneration. Neurology [Suppl] 39:260.

Gilbert CD, Wiesel TN (1979) Morphology and intracortical projections of functionally identified neurons in cat visual cortex. Nature 280:120-125.

Graus F, Cordon-Cardo C, Posner JB (1985) Neuronal antinuclear antibody in sensory neuronopathy from lung cancer. Neurology 35 : $538-543$.
Graus F, Elkon KB, Cordon-Cardo C, Posner JB (1986) Sensory neuropathy and small cell lung cancer: anti-neuronal antibody that also reacts with the tumor. Am J Med 80:45-52.

Graus F, Eldon KB, Lloberes P, Ribalta T, Torres A, Ussetti P, Valls J, Obach J, Agusti-Vidal A (1987) Neuronal antinuclear antibody (anti-Hu) in paraneoplastic encephalomyelitis. Acta Neurol Scand 75: 249-252.

Greenlee JE, Brashear HR (1983) Antibodies to cerebellar Purkinje cells in patients with paraneoplastic cerebellar degeneration and ovarian carcinoma. Ann Neurol 14:609-613.

Greenlee JE, Brashear R (1987) Immunofluorescent labeling of Purkinje and other cerebellar cell types with antibody eluted from malignant ovarian neoplasm. Ann Neurol 22:163.

Greenlee JE, Brashear HR, Herndon RM (1988) Immunoperoxidase labeling of rat brain sections with sera from patients with paraneoplastic cerebellar degeneration and systemic neoplasia. J Neuropathol Exp Neurol 47:561-571.

Gulcher JR, Stefansson K (1988) Paraneoplastic syndromes of the nervous system. Immunol Allergy Clin N Am 8:295-314.

Hsu S-M, Raine L, Fanger H (1981) Use of avidin-biotin peroxidase complex $(\mathrm{ABC})$ in immunoperoxidase techniques: a comparison beIween ABC and unlabeled antibody (PAP) procedures. J Histochem Cytochem 29:577-580.

Jaeckle KA, Graus F, Houghton A, Cordon-Cardo C, Nielsen SL, Posner JB (1985) Autoimmune response of patients with paraneoplastic cerebellar degeneration to a Purkinje cell cytoplasmic protein antigen. Ann Neurol 18:592-600.

Katz L (1987) Local circuitry of identified projection neurons in cat visual cortex brain slices. J Neurosci 7:1223-1249.

Tanaka K, Yamazaki M, Sato S, Toyoshima I, Yamamoto A, Miyatake T (1986) Antibodies to brain proteins in paraneoplastic cerebellar degeneration. Neurology 36:1169-1172.

Towbin H, Staehelin T, Gordon J (1979) Elcctrophoretic transfer of proteins from polyacrylamide gels to nitrocellulose sheets: procedure and some applications. Proc Natl Acad Sci USA 76:4350-4354.

Tsukamoto T, Yamamoto H, Iwasaki Y, Yoshie O, Terunuma H, Suzuki $H$ (1989) Antineural autoantibodies in patients with paraneoplastic cerebellar degeneration. Arch Neurol 46:1225-1229.

Vincent A, Lang B, Newsome-Davis J (1989) Autoimmunity to the voltage-gated calcium channel underlies the Lambert-Eaton myasthenic syndrome, a paraneoplastic disorder. Trends Neurosci 12:496502.

Yanagihara T, Hamberger A (1973) A method for separation of Purkinje cell and granular cell-enriched fractions from rabbit cerebellum. Brain Res 59:445-448. 